\title{
Análise espacial da vacinação contra hepatite $B$ em gestantes em área urbana no Brasil
}

\author{
Spatial analysis of vaccination against Hepatitis B in pregnant \\ women in an urban Brazilian area
}

Thales Philipe Rodrigues da Silva (https://orcid.org/0000-0002-7115-0925) ${ }^{1}$

Crizian Saar Gomes (https://orcid.org/0000-0001-6586-4561) ${ }^{1}$

Ariene Silva do Carmo (https://orcid.org/0000-0002-3421-9495) ${ }^{2}$

Larissa Loures Mendes (https://orcid.org/0000-0002-0031-3862) ${ }^{3}$

Edna Maria Rezende (https://orcid.org/0000-0002-7397-8961) ${ }^{4}$

Gustavo Velasquez-Melendez (https://orcid.org/0000-0001-8349-5042) ${ }^{4}$

Fernanda Penido Matozinhos (https://orcid.org/0000-0003-1368-4248) ${ }^{4}$
${ }^{1}$ Programa de PósGraduação em Enfermagem, Escola de Enfermagem, Universidade Federal de Minas Gerais (UFMG). Av. Alfredo Balena 190, Santa Efigênia. 30130-100 Belo Horizonte MG Brasil. thalesphilipe27 @hotmail.com

${ }^{2}$ Programa de Pós-

Graduação Saúde da Criança e Adolescente, Faculdade de Medicina, UFMG. Belo Horizonte MG Brasil.

${ }^{3}$ Departamento de Nutrição, Escola de Enfermagem, UFMG. Belo Horizonte MG Brasil.

${ }^{4}$ Departamento de Enfermagem MaternoInfantil e Saúde Pública, Escola de Enfermagem, UFMG. Belo Horizonte MG Brasil.
Abstract The objective of this article is to analyze the spatial distribution of Hepatitis $B$ vaccine (HBVAC) of pregnant women. This is a cross-sectional study carried with 266 puerperae. The $H B$ VAC record was obtained through the prenatal care booklet. The spatial scanning technique was used to detect a cluster of risk for the presence or absence of an HBVAC record. After this cluster identification, the individual and environmental variables were compared between the Coverage Areas of Basic Health Units (CAs-BHUs). The mean prevalence of non-HBVAC was $88.34 \%$. Scan spatial scan analysis observed a cluster of a high prevalence of puerperae with a HBVAC record. Comparative analyses have shown that paid work and the number of prenatal visits are positively associated with an HBVAC record. Given the above, this work brings a reflection on possible disparities with other CAs-BHUs, besides the influence of the environmental perspective. It should be emphasized that the vaccination situation is influenced not only by factors intrinsic to the individuals. However, in this study, the results indicate that individual variables are predominantly mandatory in the decision of HBVAC uptake among pregnant women.

Key words Hepatitis B, Pregnant women, Vaccination, Spatial analysis, Women's Health
Resumo O objetivo deste artigo é analisar a distribuição espacial da vacina contra Hepatite $B$ (VCHB) de gestantes. Estudo transversal, realizado com 266 puérperas. O registro da VCHB foi obtido por meio da caderneta de pré-natal. Para a detecção do cluster de risco para presença de registro ou ausência de VCHB foi utilizada a técnica de varredura espacial. Posteriormente a esta identificação do cluster, procedeu-se à comparação das variáveis individuais e ambientais entre as Áreas de Abrangência da Unidade Básica de Saúde (AA -UBS). A prevalência média de não VCHB foi de $88,34 \%$. A análise de varredura espacial Scan observou-se um cluster de alta prevalência de puérperas que apresentava o registro da VCHB em suas cadernetas. Análises comparativas demonstraram que trabalho remunerado e o número de consultas realizadas no pré-natal estão associados positivamente a VCHB. Este trabalho suscita uma reflexão de possiveis disparidades com as demais AA-UBS, além da perspectiva a nível ambiental. Ressalta-se que a situação vacinal sofre influência não somente de fatores intrinsecos aos indivíduos, entretanto, nesse estudo, os resultados apontam que as variáveis individuais são majoritariamente mandatórias na decisão das gestantes em vacinarem.

Palavras-chave Hepatite B, Gestante, Vacinação, Análise espacial, Saúde da mulher 


\section{Introdução}

A infecção pelo vírus da hepatite B (VHB) é um dos principais problemas de saúde mundial ${ }^{1,2}$. Em 2015, a prevalência mundial de infecção por VHB foi de $3,5 \%^{2}$. A Organização Mundial da Saúde (OMS) ressalta que aproximadamente 257 milhões de pessoas viviam com infecção pelo VHB e 25,3\% (65 milhões) das mulheres em idade reprodutiva estão infectados cronicamente ${ }^{2}$. Ressalta-se que mulheres em idade fértil podem transmitir o VHB para seus bebês ${ }^{3}$.

Gestantes e seus conceptos são o grupo populacional com o maior risco de complicações devido a doenças imunopreveníveis e potencialmente fatais ${ }^{4}$, pois durante o ciclo gravídico-puerperal as mulheres sofrem alterações imunológicas e fisiológicas próprias deste período, as quais predispõem as gestantes à maior susceptibilidade às infecções.

Neste contexto, os programas de imunizações contribuem para a melhoria da qualidade de vida da gestante e aumento da expectativa de vida mundial em virtude da redução, controle ou erradicação de determinadas doenças imunopreveníveis ${ }^{6}$. Ressalta-se que a vacina contra hepatite B (recombinante) (VCHB) configura-se como a maneira mais eficaz na prevenção da infecção do Vírus da Hepatite $\mathrm{B}^{7}$.

Em março de 2012, o grupo Strategic Advisory Group of Experts (SAGE) estabeleceu uma equipe de trabalho que buscou compreender os fatores determinantes da vacinação ${ }^{8}$. O grupo elaborou um Modelo organizado em três principais domínios: 1. Influências contextuais - fatores históricos, socioculturais, ambientais, do sistema de saúde/institucionais, econômicos ou políticos; 2. Influências individuais e de grupo - decorrentes da percepção pessoal da vacina ou influências do ambiente social; e 3. Questões específicas de vacinas e diretamente relacionadas as suas características ou ao processo de vacinação ${ }^{8}$. A situação vacinal sofre, portanto, influência de fatores extrínsecos aos indivíduos. Fatores contextuais (ambientais) são, também, responsáveis pelo êxito (ou não) da imunização ${ }^{9,10}$. No contexto da vacinação, níveis econômicos e socioculturais mais baixos estão associados a menores coberturas vacinais ${ }^{11}$. Entretanto, no cenário emergente mundial, há uma crescente hesitação em vacinar pessoas com elevada condições econômicas e culturais, o que se configura um problema de saúde pública ${ }^{10,12}$.

O georreferenciamento dos eventos de saúde ganha relevância na análise e avaliação de riscos à saúde coletiva, uma vez que incorpora variáveis relacionadas com o meio ambiente e com o perfil socioeconômico de uma determinada população ${ }^{13}$. A utilização das técnicas de análise espacial, otimizada por meio do processo de georreferenciamento, tem sido bastante utilizada na saúde pública, pois permite a identificação de possíveis nós-chave na propagação de determinado agravo, definida em tempo e locais geográficos específicos, além de possibilitar entender determinados episódios de doenças ou agravos e prever e planejar ações de intervenções futuras mais assertivas ${ }^{14}$.

No contexto da gestação, poucos são os trabalhos que empregam a análise espacial para verificar a distribuição espacial dos casos de ausência de registro e registro vacinal de Hepatite B em gestantes. Diante do exposto, o este estudo teve como objetivo analisar a distribuição espacial da VCHB de uma área urbana no Brasil.

\section{Métodos}

Trata-se de um estudo com delineamento transversal, desenvolvido com dados da pesquisa: "Nascer em Belo Horizonte: Inquérito sobre o parto e nascimento", que adotou os mesmos critérios da pesquisa nacional intitulada "Nascer no Brasil: Inquérito sobre o parto e nascimento"15. A coleta de dados ocorreu no período de novembro de 2011 a março de 2013 e foram incluídas as puérperas de sete maternidades que atendem à rede pública de saúde e de quatro maternidades que atendem à rede suplementar de saúde em Belo Horizonte, Minas Gerais, Brasil.

Em relação aos critérios de inclusão deste estudo, participaram todas as mulheres admitidas nas maternidades selecionadas por ocasião da realização de parto, que possuíam a caderneta de gestante no momento da sua admissão e eram residentes de Belo Horizonte. A amostra final constituiu-se de 266 puérperas.

A coleta de dados da pesquisa ocorreu por meio de entrevista às puérperas pelo menos 6 horas após o parto - sendo este tempo pré-estabelecido como intervalo mínimo definido para descanso pós-parto - e por meio de prontuário referente ao trabalho de parto e parto dessas mulheres. Esta etapa foi realizada com auxílio de questionário padronizado com variáveis de identificação, sociodemográficas, hábitos de vida, informações nutricionais, antecedentes clínicos e obstétricos, além de variáveis referentes ao plano de saúde. Ademais, durante a internação hospita- 
lar, foram fotografadas as cadernetas de pré-natal das puérperas, as quais continham os dados da vacinação das gestantes, como: vacinas aplicadas, número de dose e exames sorológicos. Ressalta-se que todas as etapas da coleta de dados foram realizadas por enfermeiros previamente treinados.

Para este estudo, a presença de informação acerca da vacinação da puérpera ou o registro de apenas uma dose da VCHB presente na caderneta de pré-natal foi utilizada como uma medida indireta da imunização da gestante e considerada como variável desfecho.

A partir do endereço da residência das participantes do estudo, foram atribuídas, a cada um desses endereços, coordenadas geográficas (latitude e longitude) e as puérperas foram alocadas nos Sistemas de Informações Geográficas (SIG). Este processo possibilitou o georreferenciamento exato das puérperas no espaço de Belo Horizonte, bem como a organização das variáveis ambientais de acordo com a Área de Abrangência da Unidade Básica de Saúde (AA-UBS). Ressaltase que o entorno físico e social das puérperas foi definido utilizando o princípio das AA-UBS, pois se inferiu que residir nessas AA-UBS significa ter características similares do entorno social e físico.

Para caracterizar as variáveis contextuais, foi elaborada uma base geocodificada com os dados ambientais selecionadas por meio de revisão da literatura ${ }^{8}$, à qual foram incorporados os dados individuais de cada participante da amostra. Esses dados foram georreferenciados a partir do endereço e CEP do local, obtidos a partir de diversas fontes comerciais e governamentais, como: Centro Integrado de Informações de Defesa Social (CINDS) da Polícia Militar de Minas Gerais, Instituto Brasileiro de Geografia e Estatística (IBGE), Secretaria Municipal de Saúde (SMS) e Empresa de Informática e Informação do Município de Belo Horizonte S/A (PRODABEL).

O geoprocessamento das variáveis ambientais baseou-se nos endereços dos setores censitários e, posteriormente, foi realizada a união pela AA-UBS de Belo Horizonte. As variáveis contextuais referem-se ao ambiente construído (densidade populacional da AA-UBS) e ao ambiente social (taxa de criminalidade da AA-UBS e Índice de Vulnerabilidade da Saúde (IVS) médio da AA-UBS).

A taxa de criminalidade foi gerada a partir de dados que incluem estupro, extorsão mediante sequestro, homicídios, roubo, sequestro e cárcere privado.

Em relação ao IVS, trata-se de um indicador sintético construído a partir de características so- cioeconômicas e demográficas e de saneamento básico de acordo com as informações do Censo do Instituto Brasileiro de Geografia e Estatística (IBGE). Deste modo, ele avalia a vulnerabilidade da cidade de Belo Horizonte espacialmente em 4 índices, sendo eles: área de baixa vulnerabilidade à saúde, área de média vulnerabilidade à saúde, área de elevada vulnerabilidade à saúde e área de muito elevada vulnerabilidade à saúde ${ }^{16}$.

Para o processo de geocodificação dos endereços, utilizou-se o pacote ggmap no $\mathrm{R}$, versão 3.4.3. A identificação da AA-UBS da puérpera deu-se por meio do programa QGIS versão 2.18.14. A descrição da população foi realizada por meio do pacote estatístico Statistical Software for Professional (Stata), versão 14.0.

Primeiramente, foi realizada a descrição da população de estudo e as estimativas foram apresentadas em proporções (\%), com Intervalo de Confiança (IC95\%). Para as variáveis quantitativas, depois de verificada a assimetria pelo teste Shapiro-Wilk, os dados foram apresentados por meio de mediana e intervalo interquartílico (IQ).

Foram construídos mapas coropléticos para verificar a distribuição espacial da prevalência de ausência de registros da vacina.

Para identificar áreas de maior concentração de ausência de registro e registro vacinal de Hepatite B em gestantes foi utilizada a técnica de Kernel. O mapa de Kernel permite estimar a quantidade de eventos por unidade de área em cada célula de uma grade regular que recobre a região estudada. Foi gerado um mapa de Kernel para ausência de registro e outro para registro vacinal de Hepatite B em gestantes utilizando-se método Normal de Estimativa (Quártica, biponderada) e tamanho do bandwidth ou raio foi fixado em 800 metros para ambos os mapas. Para esse procedimento analítico utilizou o programa QGIS versão 2.18.14.

Para a detecção do cluster de risco para presença de registro de VCHB foi utilizada a técnica de varredura espacial ${ }^{17}$. Essa técnica fundamenta-se na razão da máxima verossimilhança entre as áreas. Assim, um conglomerado de risco é um grupo de eventos, limitados geograficamente, em concentração e tamanho, e de risco distinto ${ }^{18}$. Dessa forma, a hipótese nula dessa técnica é a aleatoriedade espacial dos casos.

O nível de significância adotado foi $\alpha=0,05$ e as análises foram realizadas com o uso do Software for the spatial, Temporal and Space-time scan statistics (SaTScan), versão 9.2.

Posteriormente à identificação do cluster, procedeu-se à comparação do cluster de presen- 
ça de registro para VCHB e as demais AA-UBS entre as variáveis ambientais, por meio do teste Mann-Whitney. Para verificar possíveis diferenças entre as puérperas que pertenciam ao cluster de presença de registro para VCHB e as demais AA-UBS, utilizou-se a análise comparativa das características socioeconômicas e obstétricas. Utilizou-se, para a comparação, o teste Exato de Fisher para as variáveis categóricas e o teste Mann-Whitney para as variáveis contínuas, considerando-se como nível de significância de 5\%.

Para análise dos dados, foi utilizado o pacote estatístico Statistical Software for Professional (Stata), versão 14.0. Considerou-se um nível de significância de $\alpha<0,05$ em todos os procedimentos analíticos.

A pesquisa referente à "Vacinação de gestantes: avaliação dos aspectos epidemiológicos e clínicos no município de Belo Horizonte" foi aprovada pelo Comitê de Ética da Universidade Federal de Minas Gerais.

\section{Resultados}

A amostra final deste estudo foi constituída por 266 puérperas. Na Tabela 1, observa-se o perfil sociodemográfico e obstétrico da amostra. A mediana de idade foi de 27,5 anos (IQ 23 - 32). Ao analisar o perfil sociodemográfico das puérperas, $69,17 \%$ viviam com companheiro, $49,63 \%$ possuíam Ensino Médio Completo e 53,75\% exerciam algum tipo de trabalho remunerado. Em relação ao histórico obstétrico, 58,64\% não eram primíparas e $62,17 \%$ não tiveram abortos anteriores à gravidez do momento da pesquisa. A mediana da idade gestacional no momento do parto, a mediana foi de 39 semanas (IQ 38 - 40) (Tabela 1).

A prevalência média de ausência de registro de VCHB foi de $88,34 \%$, variando entre as AA -UBS. A Figura 1 apresenta a prevalência da ausência de registro de VCHB segundo as AA-UBS. Observou-se uma aparente distribuição homogênea da ausência de registro para VCHB de acordo com as 115 AA-UBS das UBS, variando de 0,0 a $100,00 \%$. As áreas com vermelho mais escuro revelam maior concentração de puérperas sem o registro de VCHB na caderneta das gestantes.

$\mathrm{Na}$ Figura 2, encontram-se os mapas de Kernel de ausência e presença de registro para a VCHB nas cadernetas de gestantes. Observa-se, em análise preliminar, que a probabilidade de densidade para ausência de registro para VCHB aponta uma maior prevalência não registro para
Tabela 1. Perfil sociodemográfico e obstétrico da amostra de puérperas. Belo Horizonte, 2011-2013.

\begin{tabular}{lrc}
\hline & $\mathbf{n}(\%)$ & \multicolumn{1}{c}{ IC95\% } \\
\hline $\begin{array}{l}\text { Perfil sociodemográfico } \\
\text { Idade }\end{array}$ & & \\
Estado Civil & $27,5(23-32)$ & \\
$\quad$ Vive com o companheiro & $184(69,17)$ & $63,32-74,46$ \\
$\quad$ Não vive com o & $82(30,83)$ & $25,53-36,67$ \\
$\quad$ companheiro & & \\
Escolaridade & & \\
Ensino Fundamental & $90(33,83)$ & $28,36-39,77$ \\
Ensino Médio & $132(49,62)$ & $43,61-55,64$ \\
Ensino Superior & $44(16,54)$ & $12,52-21,53$ \\
Cor de pele autorreferida & & \\
Branca & $76(28,57)$ & $23,43-34,33$ \\
$\quad$ Não branca & $190(71,43)$ & $65,66-76,56$ \\
Trabalho remunerado & & \\
$\quad$ Não & $123(46,24)$ & $40,29-52,29$ \\
Sim & $143(53,76)$ & $47,70-49,70$ \\
Perfil obstétrico & & \\
No de consultas & $9(7-10)$ & \\
realizadas no pré-natal & & \\
Primípara & & \\
Sim & $110(41,35)$ & $35,54-47,40$ \\
Não & $156(58,65)$ & $52,59-64,45$ \\
Aborto & & \\
Não & $97(62,18)$ & $54,23-69,51$ \\
Sim & $59(37,82)$ & $30,48-45,76$ \\
Idade gestacional do & $39(38-40)$ & \\
parto & & \\
\hline
\end{tabular}

Notas: ${ }^{*}$ Mediana (IQ); ${ }^{* *}$ Não branca inclui: Preta, Amarela, Oriental, Indígena, Parda e Morena; ${ }^{* *}$ Aborto em gestações anteriores.

regiões periféricas de Belo Horizonte. Em relação ao mapa de probabilidade de densidade para presença de registro para VCHB, verifica-se maior prevalência dos casos em áreas mais distantes $\mathrm{e}$ mais centrais de Belo Horizonte.

A análise de varredura espacial Scan é apresentada na Figura 3. Observou-se um cluster de alta prevalência de puérperas que apresentava $\mathrm{o}$ registro da VCHB em suas cadernetas, com evidência significativa $(\mathrm{p}=0,014)$. Ressalta-se que foi realizada a análise de varredura Scan para não registro de VCHB e não se encontrou cluster estatisticamente significativo.

Em relação ao cluster de alta prevalência para registro de VCHB, o mesmo possui um raio de $2.008,1$ metros, com $83,3 \%$ de casos de registro para VCHB nas cadernetas de gestantes e risco relativo de 8,33, demonstrando a probabilidade de encontrar um puérpera com registro $\mathrm{VCHB}$ no 
Figura 1. Distribuição espacial das prevalências de ausência de registro para VCHB (recombinante) (\%) de acordo com as AA-UBS das UBS. Belo Horizonte, 2011-2013.

Fonte: Elaborada para fins desse estudo.

cluster é 8,33 vezes comparado com as áreas fora do cluster (Tabela 2 ).

Em análise comparativa das variáveis ambientais entre o cluster de registro nas cadernetas para VCHB, não foram verificadas diferenças para as variáveis ambientais. Este aspecto demonstrou que, possivelmente, as variáveis ambientais disponíveis neste estudo não foram capazes de explicar as diferenças entre o cluster e demais AA-UBS (Tabela 3).

Por fim, foram realizadas análises comparativas das características individuais e o cluster de registro nas cadernetas para VCHB. Observou-se diferença entre o trabalho remunerado e o número de consultas realizadas no pré-natal (Tabela 4).

\section{Discusssão}

Esse trabalho analisou a distribuição espacial da VCHB em gestantes de Belo Horizonte e verifi- cou que a prevalência de não registro e, consequentemente, de não vacinação para VHB foi de $88,34 \%$, demonstrando baixa adesão por parte das gestantes para a VCHB. A baixa adesão para VCHB é um dado similar ao encontrados em outros estudos $^{19-21}$. No Brasil, trabalho realizado com gestantes adolescentes no Pará evidenciou que $70,4 \%$ não tinham recebido a $\mathrm{VCHB}^{22}$, entretanto estudo realizado com gestantes paulistas, e que considerou mais de uma vacina - além da VCHB -, encontrou uma taxa de vacinação de $68,4 \%{ }^{23}$.

Sabe-se que a VCHB é o método mais eficaz na prevenção da infecção contra o $\mathrm{VHB}^{7}$, portanto torna-se necessária maior abrangência da imunização da população gestante, a fim de aumentar as taxas de cobertura vacinal para esta população. A vacinação deve ser uma prática incorporada no cotidiano das unidades de saúde, além de ser uma ação prioritária, efetiva e estratégica da Atenção Primária à Saúde (APS) ${ }^{23}$. 


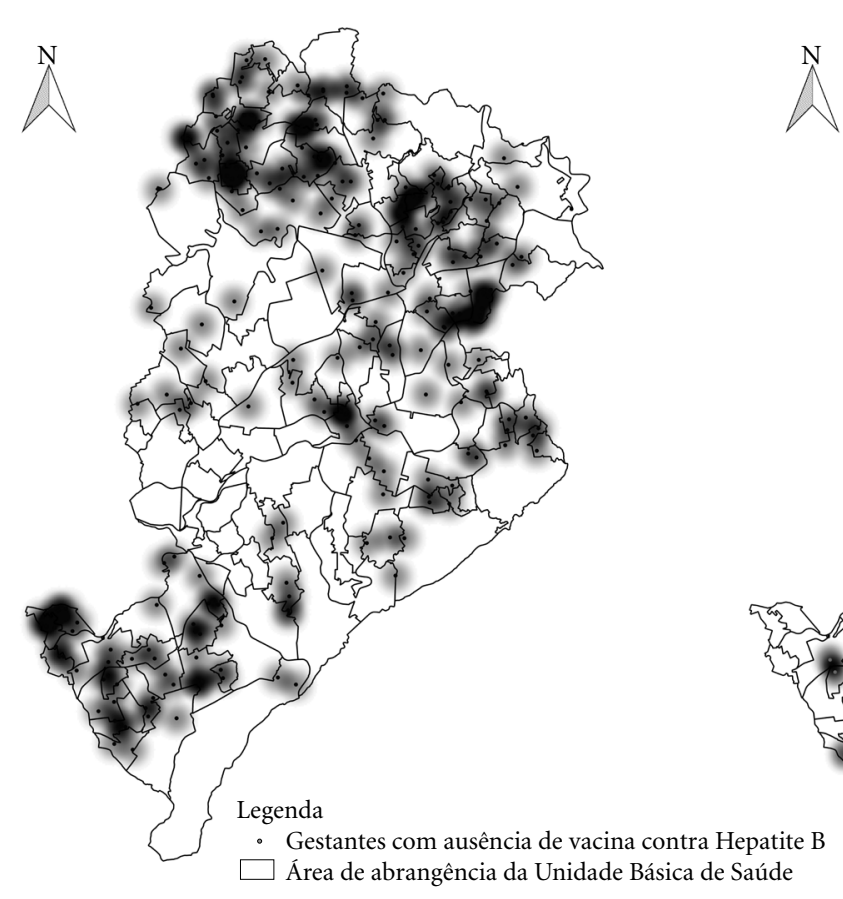

$\square$ Área de abrangência da Unidade Básica de Saúde

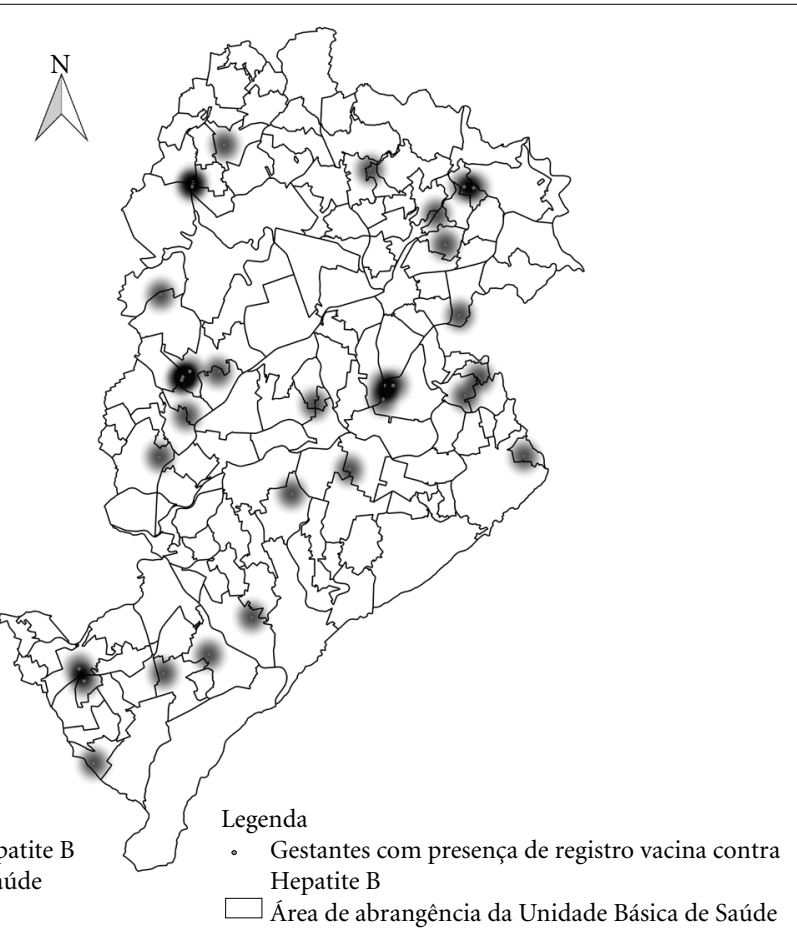

Hepatite B

$15 \quad 20 \mathrm{~km}$

Figura 2. Estimação da densidade de Kernel de ausência e presença de registro nas cadernetas de gestantes para a VCHB. Belo Horizonte, 2011-2013.

Fonte: Elaborada para fins desse estudo.

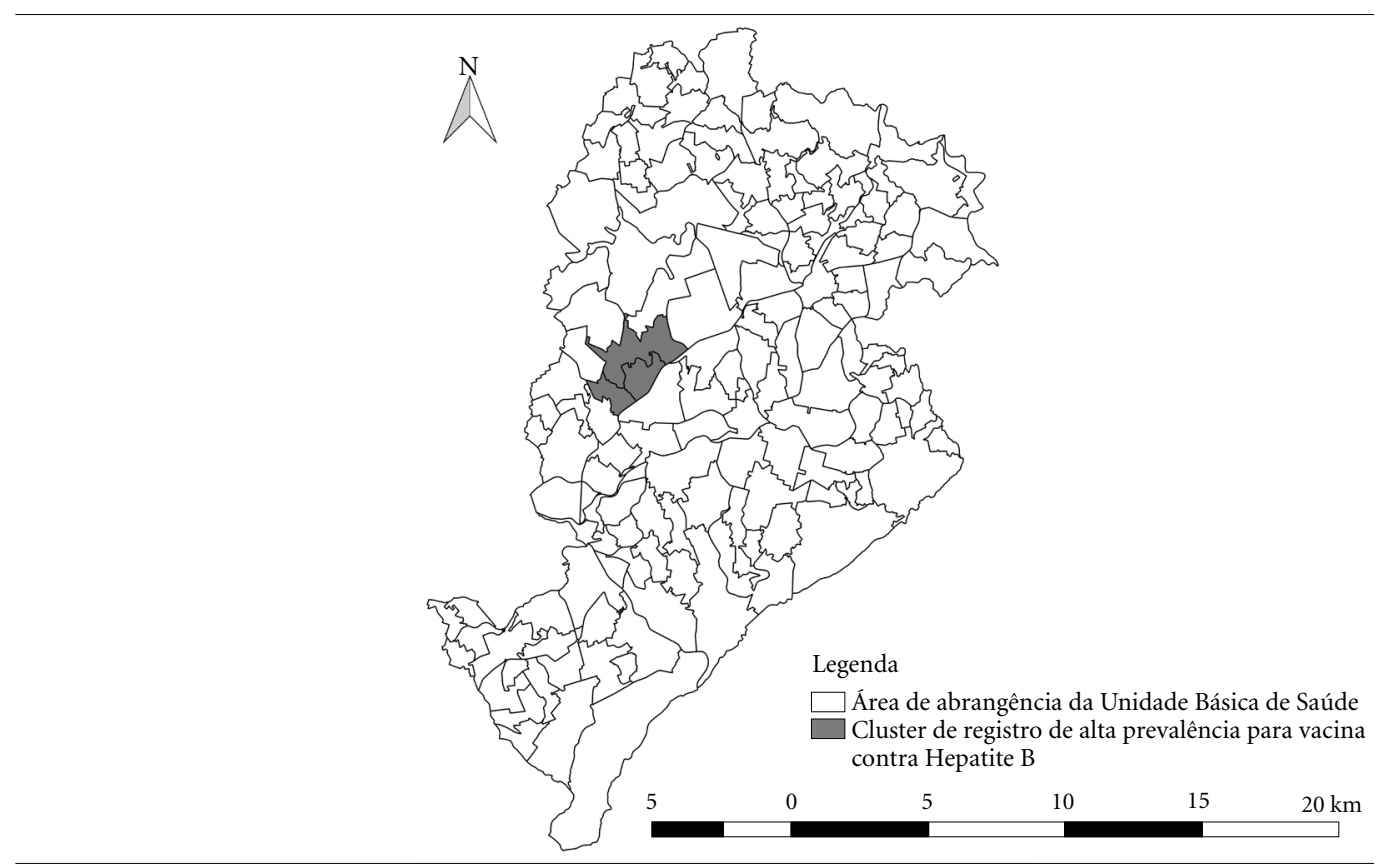

Figura 3. Análise espacial dos casos de presença de registro nas cadernetas de gestantes para a VCHB. Belo Horizonte, 2011-2013. 
Tabela 2. Perfil do cluster de alta prevalência para registro de VCHB (recombinante) nas cadernetas de gestantes. Belo Horizonte, 2011-2013.

\begin{tabular}{lc}
\hline & $\begin{array}{c}\text { Cluster de alta } \\
\text { prevalência }\end{array}$ \\
\cline { 2 - 2 } & Registro para VCHB \\
\hline Raio (m) & $2.008,10$ \\
No de pessoas no Cluster & 6 \\
\% de casos & 83,3 \\
No de Áreas de Abrangências & 3 \\
Risco Relativo & 8,33 \\
p-valor & $\mathbf{0 , 0 1 4}$ \\
\hline
\end{tabular}

Notas: $p$-valor $<0,05$ em negrito.
Em relação à probabilidade de densidade para ausência de registro para VCHB, observouse o predomínio nas regiões distantes dos centros urbano. O SAGE define que a hesitação em se vacinar pode ocorrer devido às barreiras geográficas e ao distanciamento do centro de saúde em relação ao local de moradia (ou ao acesso ao serviço de saúde dificultado $)^{8}$. Estudo demonstrou que os deslocamentos da população e a insegurança podem explicar parcialmente a hesitação das vacinas, devido à barreira geográfica imposta pela insegurança urbana ${ }^{26}$. Entretanto, cabe ressaltar que mesmo diante de uma cobertura vacinal inferior à da preconizada pela OMS, o PNI tem alcançado avanços nas coberturas vacinais, uma vez que descentraliza suas ações referentes à vacinação, estabelece parceria e participação de gestores estaduais e municipais, com consequente impacto na situação epidemiológica das doenças imunopreveníveis ${ }^{27}$.

Dentre os fatores individuais, observa-se que dentre as gestantes que residiam no cluster de registro nas cadernetas para VCHB, todas exerciam algum tipo de trabalho remunerado, demonstrando que tal característica individual influencia na presença de registro para VCHB. Exercer algum tipo de trabalho remunerado associa-se positivamente ao início precoce das consultas de pré-natal e ao maior número de consultas de prénatal, consequentes à maior chance de imunização materna ${ }^{28}$, uma vez que possibilita a identificação precoce de intervenções necessárias pelos profissionais $^{29}$.

Outro fator individual que se associou às puérperas residentes no cluster de registro nas cadernetas para VCHB foi à realização de maior número de consultas de pré-natal quando com-

Tabela 3. Descrição das características ambientais, de acordo com os clusteres de ausência e presença de registro nas cadernetas de gestantes para a VCHB. Belo Horizonte, 2011-2013.

\begin{tabular}{lrrr}
\hline \multirow{2}{*}{ Variáveis ambientais } & \multicolumn{2}{c}{ Vacina recombinante contra o vírus da Hepatite B } \\
\cline { 2 - 3 } & $\begin{array}{c}\text { Demais Áreas de } \\
\text { Abrangências }\end{array}$ & Cluster de registro & p-valor \\
\cline { 2 - 3 } & Mediana (IQ) & Mediana (IQ) & 0,563 \\
\hline $\begin{array}{l}\text { Densidade populacional } \\
\left.\text { (habitantes } / \mathrm{km}^{2}\right)\end{array}$ & $9.454,867$ & 8.311 .669 & \\
Taxa de criminalidade* & $(7.148,006-11.848,14)$ & $(4.830,148-11.360,22)$ & \\
Renda Total & $10,94(6,26-16,34)$ & $8,81(7,30-10,41)$ & 0,629 \\
& 9.498 .225 & 8.442 .414 & 0,759 \\
IVS & $(5.970 .467-20.200 .000)$ & $(7.277 .025-31.400 .000)$ & \\
\hline
\end{tabular}

Notas: Teste Mann-Whitney. IQ - Intervalo Interquartílico. IVS - Índice de Vulnerabilidade da Saúde. ${ }^{\star}$ Taxa de criminalidade por 10.000. ${ }^{* \star}$ Renda total nominal mensal de indivíduos com 10 anos ou mais de idade das AA-UBS das UBS.

Fonte: Elaborada para fins desse estudo. 
Tabela 4. Fatores individuais por conglomerados de ausência e presença de registro nas cadernetas de gestantes para a VCHB. Belo Horizonte, 2011-2013.

\begin{tabular}{|c|c|c|c|}
\hline \multirow{3}{*}{ Variáveis individuais } & \multicolumn{3}{|c|}{ Vacina recombinante contra o vírus da Hepatite B } \\
\hline & \multirow{2}{*}{$\begin{array}{c}\text { Demais Áreas de } \\
\text { Abrangência }\end{array}$} & \multirow{2}{*}{$\begin{array}{c}\text { Cluster de registro } \\
\mathbf{n}(\%)\end{array}$} & \multirow{2}{*}{$\begin{array}{c}\text { p- } \\
\text { valor }\end{array}$} \\
\hline & & & \\
\hline \multicolumn{4}{|l|}{ Socioeconômicas } \\
\hline Idade & $27(22-32)$ & $30(26-34)$ & 0,295 \\
\hline Estado Civil & & & 0,182 \\
\hline Vive com o companheiro & $178(68,46)$ & $6(100)$ & \\
\hline Não vive com o companheiro & $82(31,54)$ & 0 & \\
\hline Escolaridade & & & 0,754 \\
\hline Ensino Fundamental & $89(34,23)$ & $1(16,67)$ & \\
\hline Ensino Médio & $128(49,23)$ & $4(66,67)$ & \\
\hline Ensino Superior & $43(16,54)$ & $1(16,67)$ & \\
\hline Cor de pele autorreferida & & & 1,000 \\
\hline Branca & $47(28,46)$ & $2(33,33)$ & \\
\hline Não branca ${ }^{\star *}$ & $186(71,54)$ & $4(66,67)$ & \\
\hline Trabalho Remunerado & & & 0,032 \\
\hline Não & $123(47,31)$ & 0 & \\
\hline Sim & $137(52,69)$ & $6(100)$ & \\
\hline \multicolumn{4}{|l|}{ Histórico obstétrico } \\
\hline No de consultas realizadas no pré-natal ${ }^{\star}$ & $9(7-10)$ & $12(11-13)$ & 0,005 \\
\hline Primípara & & & 0,406 \\
\hline Sim & $109(41,92)$ & $1(16,67)$ & \\
\hline Não & $151(58,08)$ & $5(83,33)$ & \\
\hline Aborto $^{* * *}$ & & & 1,000 \\
\hline Não & $94(62,25)$ & $3(60,00)$ & \\
\hline Sim & $57(37,75)$ & $2(40,00)$ & \\
\hline Idade gestacional do parto & $39(38-40)$ & $38,5(38-39)$ & 0,451 \\
\hline
\end{tabular}

paradas às puérperas residentes nas demais $\mathrm{AA}$ -UBS. Ressalta-se que a mediana de consultas de pré-natal das mulheres deste estudo foi além da preconizada pela OMS, que recomenda um mínimo de oito $^{30}$. Entretanto, torna-se necessário ir além dos valores numéricos das consultas realizadas durante o período gestacional, deve-se levar em consideração os aspectos relativos aos conteúdos abordados nessas consultas, incluindo a atenção e o tempo despendidos e as orientações prestadas à gestante por parte dos profissionais de saúde ${ }^{31}$. O papel dos profissionais de saúde pode influenciar as mulheres em suas decisões sobre a vacinação durante a gravidez e a ausência de recomendação de vacinação, por parte dos profissionais de saúde durante o pré-natal, é um dos principais motivos para o desfecho de não vacinação ${ }^{28,32,33}$. Assume-se que é tarefa de todos os membros da equipe da APS, e especialmente do pré-natalista, verificar o estado vacinal das gestantes e, se necessário, atualizar ou iniciar o esquema vacinal ${ }^{34}$.

Ressalta-se que as variáveis ambientais disponíveis nesse estudo não demonstraram diferença estatística.

Nesse caso em particular, a ausência de significância estatística poderia ser explicada pelo número limitado de variáveis ambientais. Dessa forma, caso novas variáveis estivessem disponíveis para esse local em particular, seria possível existir maior evidência para explicar os fatores associados à VCHB.

Por fim, este estudo apresenta algumas limitações, como o fato de os dados georreferenciados das variáveis ambientais serem provenientes de fontes governamentais, podendo estar sujeitos a imprecisões. Ressalta-se que os dados utilizados foram checados por meio de outras plataformas 
de geocodificação para minimizar tal limitação. Este estudo avança na perspectiva da vacinação, uma vez que detectou um cluster de registro nas cadernetas para VCHB, identificando regiões geográficas em Belo Horizonte que possuem certa similaridade.

\section{Colaboradores}

TPR Silva participou do planejamento da pesquisa, análise estatística, interpretação dos dados, redação do artigo, revisão crítica relevante do conteúdo intelectual e aprovação da versão a ser publicada. CS Gomes e AS Carmo participaram da interpretação dos dados, redação do artigo, revisão crítica relevante do conteúdo intelectual e aprovação da versão a ser publicada. LL Mendes; EM Rezende e G Velásquez-Meléndez participaram da redação do artigo, revisão crítica relevante do conteúdo intelectual e aprovação da versão a ser publicada. FP Matozinhos foi responsável pela concepção e projeto, supervisão, redação do artigo, revisão crítica do conteúdo intelectual e aprovação da versão a ser publicada.

\section{Agradecimentos}

Ao Núcleo de Pesquisa e Estudo em Vacinação (NUPESV) da Escola de Enfermagem da UFMG.

\section{Conclusão}

Diante do exposto, este trabalho suscita uma reflexão de possíveis disparidades com as demais AA-UBS, além da perspectiva a nível ambiental. Outras evidências encontradas neste estudo sugerem que exercer trabalho remunerado e maior número de consulta são fatores determinantes para maiores proporções de registro nas cadernetas para VCHB. Ressalta-se que a situação vacinal sofre influência não somente de fatores intrínsecos aos indivíduos, entretanto, nesse estudo, os resultados apontam que as variáveis individuais são majoritariamente mandatórias na decisão das gestantes em vacinarem.

\section{Referências}

1. Ogholikhan S, Schwarz KB. Hepatitis vaccines. Vaccines 2016; 4(6):1-17.

2. World Health Organization (WHO). Global hepatitis report 2017. Genebra: WHO; 2017.

3. Ximenes RAA-UBS, Figueiredo GM, Cardoso MRA, Stein AT, Moreira RC, Coral G, Crespo D, Santos AA, Montarroyos UR, Braga MC, Pereira LMMB, Hepatitis Study Group. Population-based multicentric survey of hepatitis B infection and risk factors in the North, South, and Southeast Regions of Brazil, 10-20 years after the beginning of vaccination. Am J Trop Med Hyg 2015; 93(6):1341-1348.

4. Munoz FM, Sheffield JS, Beigi RH, Read JS, Swamy GK, Jevaji I, Rasmussen SA, Edwards KM, Fortner KB, Patel SM, Spong CY, Ault K, Heine RP, Nesin M. Research on vaccines during pregnancy: Protocol design and assessment of safety. Vaccine 2013; 31(40):42744279 .

5. Gabutti G, Conforti G, Tomasi A, Kuhdari P, Castiglia P, Prato R, Memmini S, Azzari C, Rosati GV, Bonanni P. Why, when and for what diseases pregnant and new mothers "should" be vaccinated. Hum Vaccin Immunother 2017; 13(2):283-290.

6. Perrett KP, Nolan TM. Immunization During Pregnancy: Impact on the Infant. Pediatr Drugs 2017 19(4):313-324.

7. Miglietta A, Quinten C, Lopalco PL, Duffell E. Impact of hepatitis $B$ vaccination on acute hepatitis $B$ epidemiology in European Union/European Economic Area countries, 2006 to 2014. Euro Surveill 2018 23(6):17-00278.

8. The Strategic Advisory Group of Experts (SAGE). Appendices To the Report of the Sage Working Group. Newcastle: SAGE; 2014. 
9. Barata RB, Almeida Ribeiro MCS, Moraes JC, Flannery B. Socioeconomic inequalities and vaccination coverage: Results of an immunisation coverage survey in 27 Brazilian capitals, 2007-2008. J Epidemiol Community Health 2012; 66(10):934-941.

10. Berezin M, Eads A. Risk is for the rich? Childhood vaccination resistance and a Culture of Health. Soc Sci Med 2016; 165:233-245.

11. Moraes JC, Ribeiro MCSA. Desigualdades sociais e cobertura vacinal: uso de inquéritos domiciliares. Rev Bras Epidemiol 2008; 11(Supl. 1):113-124.

12. Iriart JAB. Autonomia individual vs. proteção coletiva: a não-vacinação infantil entre camadas de maior renda/escolaridade como desafio para a saúde pública. Cad Saude Publica 2017; 33(2):2-4.

13. Nogueira MJ, Silva BFA, Barcelos SM, Schall VT. Análise da distribuição espacial da gravidez adolescente no Município de Belo Horizonte - MG. Rev Bras Epidemiol 2009; 12(3):297-312.

14. Meliker JR, Sloan CD. Spatio-temporal epidemiology: Principles and opportunities. Spat Spatiotemporal Epidemiol 2011;2(1):1-9.

15. Vasconcellos MTL, Silva PLN, Pereira APE, Schilithz AOC, Souza-Junior PRB, Szwarcwald CL. Desenho da amostra Nascer no Brasil: Pesquisa Nacional sobre Parto e Nascimento. Cad Saúde Pública 2014; 30(Supl.):S49-S58.

16. Secretaria Municipal de Saúde de Belo Horizonte. In dice de Vulnerabilidade à Saúde. Belo Horizonte; 2013.

17. Kulldorff M, Nagarwalla N. Spatial disease clusters: Detection and inference. Stat Med 1995; 14(8):799810.

18. Bavia ME, Carneiro DDMT, Cardim LL, Silva MMN, Martins MS. Estatística espacial de varredura na detecção de áreas de risco para a cisticercose bovina no estado da Bahia. Arq Bras Med Vet Zootec 2012; 64(5):1200-1208.

19. Chan OK, Suen SSH, Lao TTH, Leung VKT, Yeung SW, Leung TY. Determinants of hepatitis B vaccine uptake among pregnant Chinese women in Hong Kong. Int J Gynecol Obstet 2009; 106(3):232-235.

20. Celikel A, Ustunsoz A, Guvenc G. Determination of vaccination status of pregnant women during pregnancy and the affecting factors. J Clin Nurs 2014; 23(15-16):2142-2150.

21. Hannachi N, Bahri O, Mhalla S, Marzouk M, Sadraoui A, Belguith A, Triki H, Boukadida J. Hépatite virale B chez les femmes enceintes tunisiennes: facteurs de risque et intérêt de l'étude de la réplication virale en cas d'antigène HBe négatif. Pathol Biol 2009; 57(3):43-47.

22. Guerra AB, Siravenha LQ, Laurentino RV, Feitosa RNM, Azevedo VN, Vallinoto ACR, Ishak R, Machado LFA. Seroprevalence of HIV, HTLV, CMV, HBV and rubella virus infections in pregnant adolescents who received care in the city of Belém, Pará, Northern Brazil. BMC Pregnancy Childbirth 2018; 18(1):1-7.

23. Rocha BCC, Carvalheira APP, Ferrari AP, Tonete VLP, Duarte MTC, Parada CMGL. Cobertura vacinal e fatores associados em puérperas de município paulista. Cien Saude Colet 2016; 21(7):2287-2292.
24. Siqueira LG, Martins AMEBL, Versiani CMC, Almeida LAV, Oliveira CS, Nascimento JE, Alecrim BPA, Bezerra RC. Avaliação da organização e funcionamento das salas de vacina na Atenção Primária à Saúde em Montes Claros, Minas Gerais, 2015. Epidemiol Serv Saúde 2017; 26(3):557-568.

25. Espíndola MFS, Mesenburg MA, Silveira MF. Acesso à vacina contra a hepatite $B$ entre parturientes que realizaram o pré-natal em Pelotas, Rio Grande do Sul. Epidemiol Serv Saúde 2014; 23(3):447-454.

26. Braz RM, Domingues CMAS, Teixeira AMS, Luna EJA. Classificação de risco de transmissão de doenças imunopreveníveis a partir de indicadores de coberturas vacinais nos municípios brasileiros. Epidemiol Serv Saúde 2016; 25(4):745-754.

27. Dubé E, Gagnon D, Nickels E, Jeram S, Schuster M. Mapping vaccine hesitancy-Country-specific characteristics of a global phenomenon. Vaccine 2014; 32(49):6649-6654.

28. Araújo ACM, Guimarães MJB, Frias PG, Correia JB. Avaliação das salas de vacinação do Estado de Pernambuco no ano de 2011. Epidemiol Serviços Saúde 2013; 22(2):255-264.

29. Baum S, Hitschold T, Becker A, Smola S, Solomayer E, Rody A, et al. Implementation of the Recommendation to Vaccinate Pregnant Women against Seasonal Influenza - Vaccination Rates and Acceptance. Geburtshilfe Frauenheilkd 2017; 77(4):340-351.

30. Orgnização Mundial da Saúde (OMS). Recomendações da OMS sobre cuidados pré-natais para uma experiência positiva na gravidez. Genebra: OMS; 2016.

31. Corrêa CRH, Bonadio IC, Tsunechiro MA. Avaliação normativa do pré-natal em uma maternidade filantrópica de São Paulo. Rev Esc Enferm USP 2011; 45(6):1293-1300.

32. Nunes JT, Gomes KRO, Rodrigues MTP, Mascarenhas MDM. Qualidade da assistência pré-natal no Brasil: revisão de artigos publicados de 2005 a 2015. Cad Saúde Coletiva 2016; 24(2):252-261.

33. Krishnaswamy S, Cheng AC, Wallace EM, Buttery J, Giles ML. Understanding the barriers to uptake of antenatal vaccination by women from culturally and linguistically diverse backgrounds: A cross-sectional study. Hum Vaccines Immunother 2018; 5515:1-8.

34. Chan OK, Lao TT, Suen SSH, Lau TK, Leung TY. Knowledge on hepatitis B infection among pregnant women in a high endemicity area. Patient Educ Couns 2011; 85(3):516-520.

Artigo apresentado em 05/10/2018

Aprovado em 05/06/2019

Versão final apresentada em 07/06/2019

Editores-chefes: Romeu Gomes, Antônio Augusto Moura da Silva 